Revista de Investigación Educativa 25

julio-diciembre, 2017 | ISSN 1870-5308 | Xalapa, Veracruz

Instituto de Investigaciones en Educación | Universidad Veracruzana

\title{
Adquisición de las consonantes líquidas en el español de México
}

\section{Acquisition of liquid consonants in Mexican Spanish}

\author{
Dra. Minerva Oropeza Escobar \\ Docente \\ Centro de Investigación y Estudios Superiores en \\ Antropología Social-Golfo, México \\ esmioro@yahoo.com.mx
}

El presente trabajo se centra en la adquisición de las líquidas en niños mexicanos de dos a seis años de edad cuya primera lengua es el español. En particular, se analiza el desarrollo segmental, identificando y discutiendo las diferencias individuales y sus respectivas motivaciones. Se observa que el reanálisis de los lindes morfológicos y el tratamiento analógico de secuencias fonológicas juegan un papel importante en la distribución de las líquidas en la representación fonológica del niño constituyen una fuente de diferencias a nivel de realización fonética entre niños y adultos. Asimismo, se aplican dichos hallazgos a la mejor comprensión del desarrollo atípico del lenguaje y a la logopedia.

Palabras clave: Desarrollo del lenguaje; lenguaje oral; problemas del lenguaje; terapia del lenguaje; español.

The acquisition of Spanish liquids is studied in Mexican children between the ages of two and six. Liquids are analyzed both segmentally, and in their interaction with the context in which they occur. Individual differences in the acquisition of liquids are identified, and their motivations discussed. The reanalysis of morphological boundaries and the analogical treatment of phonological sequences are shown to play a role in the distribution of liquids in the child's input, and to be a source of output-differences between children and adults. These findings are helpful to a better understanding of the atypical language acquisition and logopedia.

Keywords: Language development; spoken language; language problems; speech therapy; spanish. 


\title{
Adquisición de las consonantes líquidas en el español de México
}

\author{
Acquisition of liquid consonants in Mexican Spanish
}

\section{Introducción}

la fecha, se han realizado diversos estudios sobre la adquisición de la fonología
del español como lengua materna. Ellos difieren en varios respectos, ya sea en el eje de la investigación, la perspectiva teórica adoptada, la metodología, los datos en los que se sustenta el análisis o la variante dialectal abordada.

Así, mientras que algunos presentan una visión panorámica de la adquisición de la fonología del español (Amador, 1978; Fontanella, 1981), otros se centran en una clase específica de sonidos o contextos fonéticos (Eblen, 1980; Macken, 1975, 1978; Oropeza, 1987; Stoel, 1974). Algunos de ellos se basan en el habla espontánea (Lleó \& Prinz, 1997); otros, en datos obtenidos mediante repetición (González, 1981), en tanto que la mayoría recurre a una combinación de ambos procedimientos. Se ha dado seguimiento al proceso de adquisición ya sea en un mismo individuo a lo largo de un período específico de tiempo (Fontanella, 1981; Macken \& Barton, 1980; De Zuluaga, 1981), en varios sujetos de diferentes edades dentro de un rango específico (Amador, 1978; Bosch, 1984; Eblen, 1980; Oropeza, 1987, 2000, 2001a, 2001b), o bien siguiendo el desarrollo fonológico de varios niños durante cierto tiempo (Amador, 1978; Maez, 1981; Stoel, 1974).

Desde la perspectiva de la fonología fonéticamente orientada (phoneticallydriven-phonology) (Hayes, 1997), existen algunos trabajos sobre el español basados en restricciones que guardan relación con los grupos consonánticos homosilábicos y heterosilábicos en la población adulta (Bradley \& Schmeiser 2003; Bakovic, 2001, respectivamente). Sin embargo, existe aún un vacío en cuanto a la adquisición de la fonología de dicha lengua. El presente artículo constituye un paso en esa dirección, al centrarse en la adquisición de las líquidas (l, r y r r).

El enfoque teórico que nos ocupa busca cerrar la brecha entre la fonética — que trata con fenómenos graduales y variables - y la fonología — que se ocupa más bien de fenómenos categóricos y menos variables (Gafos, 2002; Hayes, 1997). 
Adoptando una perspectiva funcional, el enfoque de la fonología fonéticamente orientada concibe los patrones sonoros de las lenguas como efectivamente dispuestos para facilitar a) la articulación y b) la distintividad de formas contrastantes en términos de percepción (Hayes, 1997). El presente estudio, si bien se apoya en los hallazgos de los trabajos de orientación perceptual (Jun, 1995; Steriade, 1995, 2001), es principalmente de corte articulatorio. Dicha elección es coherente con el hecho de que la mayoría de la literatura disponible para los sonidos y grupos de sonidos relevantes para el presente análisis es de índole articulatoria, ya se trate del habla adulta o de la adquisición del lenguaje. Consecuentemente, las nociones "facilidad articulatoria" o "naturalidad” desempeñan un papel importante. Dichos términos han sido tradicionalmente entendidos a partir de restricciones en el habla tanto a nivel de producción como de percepción, o bien a partir del equilibrio óptimo entre demandas de percepción y articulación que compiten entre sí. Si bien podemos hablar de facilidad de articulación en general, el término se vuelve operativo en cuanto se determinan las restricciones específicas de una clase particular de sonidos. En este sentido, lo importante es no sólo el grado de dificultad inherente a la producción y mantenimiento de un estado articulatorio o acústico particular, sino, asimismo, el contexto específico en el cual ocurre un segmento (Westbury \& Keating, 1986, p. 149).

Así, la naturalidad de un sonido o clase específica de sonidos depende parcialmente de su posición al interior de la emisión en su conjunto. Este postulado encuentra soporte en el hecho de que los procesos fonológicos interlingüísticamente documentados, tales como la neutralización y la asimilación, tienen lugar en aquellos contextos en los que un sonido o secuencia implica un esfuerzo articulatorio mayor (Westbury \& Keating, 1986, pp. 160-161). Incluso, las tendencias interlingüísticas asociadas a las características universales del sistema motor interactúan con hechos fonológicos lingüísticamente específicos, tales como las dimensiones del inventario y la distribución de fonemas en el espacio acústico/articulatorio (Farnetani, 1997, p. 393). Fenómenos tales como la coarticulación, ${ }^{1}$ los patrones de coordinación y la asimilación en los grupos consonánticos han demostrado ser, en gran medida, de naturaleza lingüísticamente específica (Farnetani, 1997, p. 393 y 402; Gafos, 2002, pp. 281-282), siendo ésta la razón por la que la influencia de la fonética en la fonología no se considera tan directa,

1. En un sentido muy general, el término "coarticulación" se refiere "al hecho de que un segmento fonológico no se realiza de manera idéntica en todos los contextos, sino que a menudo varía para tornarse más similar a un segmento adyacente o cercano" (Kühnert \& Nolan, 1999, p. 7) (Traducción propia). 
sino más bien mediada por restricciones estructurales lingüísticamente específicas que se encuentran bajo cierta presión hacia la simplicidad y la simetría formal.

El conjunto de dichos factores interactúa en la adquisición de la fonología. Esto es, dado que el niño, de hecho, opera su propio aparato perceptual y de producción, es plausible mantener que tiene acceso directo al grado de dificultad articulatoria y de confusión potencial de diversas señales acústicas. Para poder determinar la dificultad de una configuración fonológica potencial, los niños recurren a medidas tales como la máxima fuerza articulatoria requerida para realizar la configuración o bien al gasto de energía (Hayes, 1997). Las condiciones lingüísticamente específicas también desempeñan un papel, puesto que las restricciones fonéticamente sustentadas pero inapropiadas para la lengua que se está aprendiendo, serán descartadas de inmediato. Por el contrario, aquéllas restricciones que son operativas en la lengua en cuestión, serán finalmente incorporadas (Hayes, 1997).

Una de las características de la adquisición de la fonología es que, en el proceso de facilitar la articulación para ajustar las configuraciones fonológicas complejas del adulto a sus aún limitadas habilidades articulatorias, el niño actúa de manera creativa y desarrolla su propia fonología. Dicho de otra manera, pone en juego restricciones y procesos fonológicos que, en sentido estricto, no corresponden a los del adulto. Pero, por otra parte, dichos procesos se encuentran representados en la fonología de otras lenguas, de tal manera que parecen basarse en un conjunto de recursos fonológicos universales.

La misma observación es válida para el proceso de adquisición en distintos individuos. Las investigaciones centradas en el proceso de adquisición han demostrado que, aun tratándose del mismo sistema fonológico, los niños, de hecho, desarrollan diferentes estrategias (Anderson \& Smith, 1987; Eblen, 1980; Lleó \& Prinz, 1996; Maez, 1981; McLeod, Van Doorn \& Reed, 2001; Stoel, 1974, entre otros). En congruencia con las observaciones precedentes, mi propósito es demostrar que el interjuego entre tendencias universales, condiciones específicas de la lengua y variación intersubjetiva, desempeñan un papel crucial en la adquisición de las líquidas del español.

Incluso cuando mi atención se ubica en los segmentos líquidos y en las secuencias consonánticas que incluyen líquidas, mi mayor preocupación por el momento es la compleja interacción de factores que intervienen en la adquisición de los sonidos líquidos.

Para dicho propósito, considero los segmentos líquidos del español en relación con las secuencias más amplias en las que ocurren. En el análisis que a continuación 
presento, el contexto desempeña un papel central en la adquisición de las líquidas, ya sea que se trate de su localización con respecto a la sílaba o unidad léxica, o bien de la naturaleza fonética de los segmentos circundantes.

Otra aportación del presente artículo es abrir a la discusión el hecho de que no sólo es posible encontrar diferentes realizaciones en diferentes sujetos. Por lo que respecta a los segmentos líquidos del español, es preciso reconocer que, para alcanzar una explicación fundamentada de su proceso de adquisición, en casos específicos existen diferentes representaciones. Se expondrán y discutirán casos de análisis morfosintáctico y fonológico basados en patrones fonotácticos sumamente frecuentes, que dan cuenta de diferencias, a nivel de producción, que difícilmente pueden sustentarse en una forma o representación fonológica igual a la del adulto. Esta observación es congruente con los hallazgos de otros estudios que atienden a la interacción entre factores tales como la morfología, la sintaxis, la gramática, el léxico y la semántica, así como la pragmática, para explicar diferencias individuales (Crystal, 1987). De hecho, desde la perspectiva de la fonología fonéticamente orientada, factores no fonéticos tales como la fidelidad ${ }^{2}$ y las restricciones en la uniformidad paradigmática, en conjunto con la tarea de adquirir las representaciones fonológicas del léxico, han desempeñado un papel importante en la adquisición de la fonología. Desde esta perspectiva se discute, asimismo, el desarrollo fonológico atípico en niños en edad escolar.

\section{Antecedentes}

Si bien son varios los estudios relevantes para mi análisis, en lo subsecuente centro mi atención en cuatro de ellos que se han enfocado en el estudio de las líquidas del español. El trabajo de Stoel (1974) es un estudio longitudinal realizado en California con quince niños que adquieren el español como primera lengua.

Una muestra complementaria para el mismo estudio fue obtenida en la Ciudad de México con niños cuyas edades fluctuaban entre 1.6 y 4.8 años, con el propósito de determinar la posible influencia del inglés en la fonología de los sujetos californianos.

2. En la teoría de la optimalidad (optimality theory), el término "fidelidad" describe la relación ideal entre la representación fonológica (input) y la realización fonética (output). Las restricciones de fidelidad impiden la disparidad entre ambas representaciones; es decir, maximizan la similitud entre ellas. En este sentido, si dos formas subyacentes distintas se producen de manera idéntica a nivel superficial, al menos una de ellas — se considera — viola la restricción de fidelidad. 
Un estudio posterior (Eblen, 1980) centrado en la adquisición de las líquidas por niños de mayor edad hablantes de español (4;0 a 5;0), fue realizado en la Ciudad de Oaxaca, México. Los datos consisten en palabras obtenidas a través de dibujos, así como en oraciones reales obtenidas mediante repetición.

Otro estudio realizado por Amador (1978) en la Ciudad de México, consideró tanto el sistema vocálico como el consonántico durante un periodo de tres meses en niños de entre 2;00 y 2;10 años de edad. Todos los sujetos mostraron formas variables para aquellas palabras que contenían las vibrantes simple y múltiple, de modo que a veces dichos sonidos se pronunciaban como tales, y a veces como $[\mathrm{d}] \mathrm{o}[\mathrm{\gamma}]$ dependiendo del contexto.

Respecto a los grupos consonánticos que incluyen líquidas, se encontró alternancia entre reducción (o supresión) y sustitución (o simplificación) de la líquida. Se identificó variación alofónica entre las líquidas laterales y las fricativas sonoras en contextos preconsonánticos y postconsonánticos, lo que constituye una característica de algunos sectores de la población de la Ciudad de México.

Por su parte, Oropeza (1987) analizó, desde una perspectiva generativista, el sistema consonántico en niños cuyas edades fluctuaban entre los 2;0 y los 6;6 años. Bernhardt et al. (2015) discuten la estructura de las palabras de niños de educación preescolar y hacen una comparación entre aquéllos que presentan un desarrollo típico y los que exhiben desarrollo atípico, incursionando también en el nivel fonológico.

Se identificaron distintos tratamientos entre diferentes individuos para grupos consonánticos específicos, ya que algunos niños son más sensibles a la estructura silábica, en tanto que otros privilegian las propiedades fonológicas de la secuencia en la que ocurren las líquidas.

\section{Objetivos}

Realizar un estudio sobre adquisición típica de las consonantes del español de México, a partir de estudios realizados por Oropeza (1989; 2000; 2001a; 2001b; 2012) en niños de 2;0 y 6:0 años y establecer una comparación con niños con desarrollo atípico a partir de valoraciones fonológicas realizadas con niños en edad escolar. 


\section{Datos y Metodología}

La autora realizó un estudio sobre fonología en niños que adquieren el español como lengua materna. Los sujetos fueron 55 niños monolingües de entre 2;0 y 6;0 años, residentes en la Ciudad de Xalapa, Veracruz. Los niños son hijos de conocidos de la investigadora. Las sesiones se grabaron y transcribieron posteriormente. Se contó con la colaboración de una colega que mostraba a los niños los materiales que a continuación se describen, en tanto que la autora del presente artículo realizaba un registro fonético simultáneo, que posteriormente se compararía con las grabaciones.

Los recursos para la obtención de la muestra fueron diseñados de tal modo que permitieran al investigador explorar cada fonema en diferentes contextos fonéticos; es decir, en posición inicial y final de palabra, así como en posición intervocálica; antes y después de consonantes específicas, y así sucesivamente. El conjunto de palabras así seleccionado, no sólo contenía los fonemas en los contextos fonéticos relevantes, sino que se pretendía que fueran fácilmente identificables por niños de corta edad. El propósito era obtener las palabras previstas, de la manera más espontánea posible. Para dicho efecto, se eligió un conjunto de imágenes que representaran el referente de cada palabra. Las imágenes se dispusieron en un álbum fotográfico, el cual se mostraba a los participantes. El material adicional consistía en un juego de memoria similar al bingo, así como un pequeño libro con escenas cotidianas para un niño mexicano del medio urbano, el cual se empleaba únicamente cuando el niño se mostraba deseoso de continuar participando. La lotería y el juego de memoria incluían palabras seleccionadas conforme a los criterios anteriores. Así, la expectativa era que los niños emitieran palabras o frases nominales. El pequeño libro invitaba más bien a realizar una descripción de los diversos objetos y actividades contenidos en cada dibujo; aunque algunos sujetos, de hecho, estructuraron un relato.

Cuando no era posible obtener una respuesta espontánea; es decir, cuando el niño encontraba difícil ya sea reconocer el objeto representado por el dibujo o bien dar con el nombre respectivo, el investigador o su asistente mencionaban la palabra y luego mostraban de nuevo la imagen al niño para que emitiera la forma esperada. 


\section{Análisis}

\subsection{Representación fonológica de las unidades léxicas que incluyen líquidas}

La naturaleza de las representaciones fonológicas —o formas "input" — en la adquisición del lenguaje, ha sido un tema recurrente de investigación. Dos posiciones han sido mantenidas por diferentes lingüistas. Algunos de ellos consideran que las representaciones adultas e infantiles difieren debido a que la percepción del niño es incorrecta o imperfecta (Braine, 1976; Macken, 1978; Vihman, 1981), o no contiene los mecanismos específicos de la lengua en cuestión que se requieren para alcanzar una representación completa (Fee, 1995, p. 60). La segunda postura mantiene que la percepción del niño se encuentra adelantada con relación a sus habilidades de producción (Ingram, 1976; Menn, 1978; Smith, 1993) y, por lo tanto, sus representaciones son similares a las del adulto. En la perspectiva de las teorías basadas en restricciones, el problema no se ha discutido a profundidad, especialmente a partir de estudios empíricos; de tal modo que las investigaciones centradas en el proceso de adquisición dan por sentado que las representaciones del niño y las del adulto son iguales.

Adoptando una perspectiva crítica, Vellem y Vihman (2002, p. 12) reconocen que el asumir que únicamente se archiva una forma, plenamente equiparable a la forma producida por el adulto, simplifica excesivamente el problema. Si bien no es posible lograr acceso a la forma exacta interiorizada por el niño para cada unidad léxica, en casos específicos es posible identificar, con cierto grado de precisión, algunas diferencias entre la forma del niño y la del adulto.

Con relación a la adquisición del español, los datos revelan que la forma interior puede diferir en cierta medida de la forma adulta respectiva. La fuente de dichas diferencias es el análisis de secuencias morfológicas potencialmente ambiguas, así como el tratamiento de las formas léxicas que responden a patrones poco frecuentes.

El primer caso que presento guarda relación con la ubicación del linde léxico entre el artículo masculino él 'él' y el sustantivo subsecuente cuando éste inicia con vocal. El reanálisis, entendido como un mecanismo que cambia la estructura subyacente de un patrón sintáctico sin implicar una modificación inmediata o intrínseca a nivel superficial (Harris \& Cambpell, 1995; Hopper \& Traugott, 2000; Timberlake, 1977), también resulta útil para entender el tratamiento de frases nominales que incluyen artículo definido o indefinido, y sustantivo. En tales casos, el límite entre el artículo y el sustantivo se reubica, afectando con ello la forma fonológica y, en consecuencia, la 
forma fonética del sustantivo correspondiente. Si dos consonantes idénticas ocurren en virtud de que el sustantivo inicia con la misma consonante con la que termina el artículo, una de ellas se elide. Secuencias tales como el oso, silabificadas por los adultos como e $\$$ lo\$so fueron frecuentemente (re)interpretadas por los niños como el loso, como si el sustantivo empezara con /1/. En estos casos, el tratamiento fonológico del niño claramente difiere de la salida (output) del adulto.

El tratamiento de los segmentos que ocurren en contextos inusuales en español también puede conllevar diferencias a nivel interno (input) entre distintos niños, por un lado, y entre el niño y el adulto, por otro.

Las características específicas de la lengua española dificultan aún más la identificación, puesto que las oclusivas y las fricativas (con excepción de [s]) son poco comunes en posición final de palabra. Al explorar mis datos encontré dos tendencias no documentadas en la población, que pueden atribuirse a la analogía. La primera consiste en reemplazar el segmento inusual por otro que, aunque no es fonéticamente afín, aparece regularmente en el mismo contexto fonotáctico o posición silábica. En tal caso, la consonante no-resonante se sustituye por la lateral o bien por la vibrante simple. ${ }^{3}$

En virtud de estas consideraciones, todo parece indicar que este fenómeno es de índole analógica, dado que tiene un efecto "regularizador", por así decir.

Debido al impacto de la analogía, la distribución de las líquidas - y de algunos otros sonidos - puede extenderse más allá de la distribución adulta y, lo que es más importante para el presente estudio, las formas resultantes se rigen por restricciones que de otra manera sería difícil asociar con estos datos específicos. Así, por ejemplo, palabras tales como /óso/ 'oso', /ílo/ 'hilo' son interpretadas por el niño como /lóso/, y /lílo/, respectivamente, y, en consecuencia, el segmento líquido /1/ es tratado como si apareciera en posición inicial. Formas adultas como /řobót/ 'robot', / kasét/ 'cassette’ y /fáb/ 'fab' (un tipo de detergente en polvo), son interpretadas como si incluyeran la vibrante simple en posición final de palabra; es decir, como /řobór/, /kasér/ y /fár/, respectivamente, $\mathrm{y}$ tratadas consecuentemente desde un punto de vista fonético.

Aun cuando, como se mencionó anteriormente, determinar la forma subyacente del niño parece una tarea inalcanzable, las evidencias disponibles, aunque limitadas

3. Se entiende por analogía, la tendencia a reemplazar una forma o estructura lingüística de distribución restringida, por una más general (Kurylowicz, 1945, citado en Hopper \&Traugott, 2000, p. 57). En otras palabras, la analogía consiste en atraer las formas menos comunes hacia las más comunes, por ejemplo, reemplazando un segmento inusual en cierto contexto, por otro que, aunque no es fonéticamente muy cercano, ocurre regularmente en dicho contexto o posición silábica (cf. Oropeza, 2012). 
a un número relativamente reducido de formas fonéticas, ayudan al analista a lograr una mayor comprensión de la interacción de factores que, operando a diferentes niveles, dan como resultado la realización fonética del niño. En los casos en que existe evidencia empírica disponible, podemos postular, con seguridad, diferentes representaciones fonológicas entre el niño y el adulto. Por lo demás, asumir una coincidencia entre ambas formas fonológicas como punto de partida, parece metodológicamente justificable.

\section{2 La adquisición de las líquidas del español}

De conformidad con la perspectiva teórica adoptada, el desarrollo de las líquidas del español se considera resultado de restricciones fonéticamente orientadas, las cuales obedecen a los criterios de simetría y simplicidad. Se demostrarán aquí que dos dimensiones que interactúan entre sí son relevantes para el análisis que nos ocupa: a) el segmento y b) el contexto en el que ocurre. Las restricciones son, en consecuencia, de naturaleza segmental o secuencial - aunque el primer tipo puede considerarse una abstracción, dado que ningún segmento ocurre fuera de cierto contexto- - Si bien no me concierne por el momento discutir el papel de la sílaba en la adquisición de la fonología, al examinar mis datos observo que el orden de adquisición de las líquidas en diferentes contextos dentro de la palabra parece regirse más por factores intersegmentales y posicionales, que por el papel de dichos sonidos como arranque o coda silábica. Examinemos cada una de ellas con mayor detenimiento.

\subsection{Restricciones segmentales y posicionales en la adquisición de la líquidas del español}

Mis datos indican que la adquisición de las consonantes del español ni es fortuita ni tiene lugar simultáneamente en diferentes posiciones de la palabra, sino que sigue un orden discernible. Las siguientes tendencias fueron identificadas en mis datos. El primer contexto en el que se adquieren las líquidas es entre vocales. En los ejemplos de la Tabla 1 podemos observar a los segmentos líquidos simplificados intervocálicamente, pero elididos a inicio de palabra. Esta situación indica, a mi juicio, distintos niveles de desarrollo del mismo segmento en distintos contextos. ${ }^{4}$

4. En el presente trabajo, se emplea el término "reducción" como sinónimo de supresión o elisión, y "simplificación” como equivalente a sustitución. 


\section{Tabla 1. Procesos fonológicos de las líquidas en posición intervocálica $\left(2 ; 0.20^{5}\right)$}

\begin{tabular}{cccc}
\hline & Forma de entrada & Forma de salida & Glosa \\
\hline 1 & /pélo/ & [péyo] & 'pelo' \\
\hline 2 & /xářa/ & [áya] & 'jarra' \\
\hline 3 & /péra/ & [péya] & 'pera' \\
\hline 4 & /calór/ & [táyo] & 'calor' \\
\hline 5 & /řelóx/ & [eyó] & 'reloj' \\
\hline 6 & /golpe/ & [ópe] & 'golpe' \\
\hline 7 & /árbol/ & [áßo] & 'árbol' \\
\hline
\end{tabular}

Fuente: Elaboración propia.

En esta etapa del desarrollo, el niño sólo produce nasales no posteriores, oclusivas $y$ africadas en posición inicial de palabra; cualquier otra consonante que ocurra en dicha posición, es suprimida o reducida. En posición intervocálica, sin embargo, las líquidas — así como otros sonidos consonánticos — se simplifican; es decir, se sustituyen por otro sonido que difiere en su especificación superficial en uno o más rasgos con respecto a la forma interna o representación subyacente (input form).

A diferencia de las líquidas en posición intervocálica - las cuales se simplifican, es decir, se sustituyen - las líquidas que ocurren antes de consonante en posición final de palabra y en posición inicial de palabra (formas 5 y 6), se reducen. ${ }^{6}$ Estos datos muestran que los segmentos consonánticos intervocálicos se adquieren primero. $\mathrm{Si}$ bien en esta temprana etapa de desarrollo las líquidas en posición inicial de palabra no son tratadas de manera diferente a las líquidas que aparecen en otros contextos; una vez que la lateral se adquiere, su uso se hace extensivo a inicio de palabra, antes que a la posición final de palabra, como lo atestiguan los datos de la Tabla 2.

5. En lo sucesivo, la edad de los niños que participaron en la presente investigación se representa en años, meses y días, como en el siguiente ejemplo: $3 ; 11.18$, que significa que el niño tiene tres años, once meses y dieciocho días.

6. Como puede apreciarse a lo largo del análisis, la edad de los niños no puede verse como un indicador contundente del nivel de desarrollo, puesto que sujetos de menor edad pueden exhibir formas fonéticas más avanzadas que la de niños de mayor edad. 
Tabla 2. Procesos fonológicos de las líquidas en posición inicial (2;0.3)

\begin{tabular}{cccc}
\hline & Forma interna & Forma externa & Glosa \\
\hline 8 & /leče/ & [leče] & 'leche' \\
\hline 9 & /paxaríto/ & [pakalíto] & 'pajarito' \\
\hline 10 & /frixól/ & [pikó] & 'frijol' \\
\hline
\end{tabular}

Fuente: Elaboración propia.

El hecho de que la lateral se adquiera antes que las vibrantes simple y múltiple, 7 se encuentra fundamentado desde un punto de vista articulatorio. A diferencia de la lateral, que implica un contacto completo y permanente con la región alveolar durante su producción, las vibrantes simple y múltiple requieren movimientos más sutiles de la lengua. La vibrante simple se articula mediante un gesto único y momentáneo, en tanto que la múltiple impone requerimientos precisos en el posicionamiento del cuerpo de la lengua.

Mis datos revelan que la posición final de palabra es el siguiente contexto en el que se manifiestan las consonantes líquidas en la adquisición de la fonología del español. Pero este tratamiento es más una tendencia que un orden fijo de extensión de los segmentos recién adquiridos.

La posición intervocálica difiere de otras posiciones silábicas, incluyendo la posición inicial de palabra, lo cual es congruente con la aseveración de Steriade (1995, 2001) en el sentido de que recurrir a la sílaba no ayuda a la comprensión de algunos fenómenos secuenciales como la neutralización y asimilación del punto de articulación. Desde este punto de vista, la posición intervocálica y la posición inicial de palabra —las cuales comparten la característica de ser arranques silábicos- pueden ser diferentes, en el caso de las líquidas, desde una perspectiva estrictamente fonética. Lo mismo puede decirse de los sonidos que actúan como "coda", ya que "final de palabra" y "antes de consonante en un grupo heterosilábico" también pueden diferir. Estudios de orientación articulatoria demuestran que la posición intervocálica es el contexto óptimo para las oclusivas sonoras, en tanto que los contextos "inicial absoluto" y "final absoluto" son más favorables para las oclusivas sordas, debido a que implican una presión subglotal menor (Westbury \& Keating, 1986). ${ }^{8}$

7. Raymond (2017, p. 15) encuentra que las líquidas $/ 1 /$ y $/ \mathrm{r} /$ se adquieren antes que $/ \check{\mathrm{r}} /$.

8. Existe, además, evidencia experimental de que las oclusivas son más difíciles de realizar después de una obstruyente que en posición inicial, y que son más fácilmente producidas después de nasal que después de otras resonantes (Hayes, 1997, p. 17). 
Que el contexto intervocálico es especial con relación a otros contextos lo sustenta la existencia de diferencias cualitativas en el tratamiento fonético que dan los niños al mismo segmento en diferentes posiciones. Como se discutirá más abajo, las elisiones son más frecuentes en las posiciones inicial de palabra, final de palabra y antes de consonante, que entre vocales. En cambio, el alargamiento compensatorio - entre otros procesos - es exclusivo de los dos últimos contextos. Considero que uno de los factores que pueden ser responsables de este tratamiento, que privilegia la más temprana presencia de las líquidas en posición intervocálica, es la sonoridad. Si bien las líquidas se caracterizan como sonidos naturalmente sonoros, parece que, en el caso de los niños de menor edad, las posiciones inicial y final implican un mayor grado de dificultad que entre vocales, donde los dos sonidos adyacentes son resonantes no consonánticos. ${ }^{9}$

Centrándonos ahora en el comportamiento de las líquidas en posición final de palabra y en interior de palabra ante consonante, encuentro que las líquidas que ocurren en los límites de palabra, se dominan antes que las líquidas preconsonánticas. Los datos de la Tabla 3 ilustran esta situación.

\section{Tabla 3. Comportamiento de las líquidas en posición final de palabra $(3 ; 11.18)$}

\begin{tabular}{cccc}
\hline & Forma interna & Forma externa & Glosa \\
\hline 11 & /árbol/ & [áßol] & 'árbol' \\
\hline 12 & /pulpo/ & [púp:o] & 'pulpo' \\
\hline 13 & /albérka/ & [aßéka] & 'alberca' \\
\hline 14 & $/$ pórke/ & {$[$ pólke] } & 'porque' \\
\hline
\end{tabular}

Fuente: Elaboración propia.

Todo parece indicar que la motivación fonética de dicho tratamiento es que los contextos interiores de palabra implican coarticulación con los segmentos adyacentes,

9. Este comportamiento no necesariamente afecta a todos los sonidos resonantes. Recordemos que las nasales se adquieren antes que las líquidas, y en el rango de edad que estudio, han sido adquiridas en todos los contextos en los que ocurren en español, excepto en grupos consonánticos entre los niños más pequeños. 
mientras que los mismos segmentos en posición inicial y final de palabra se coarticulan sólo con la vocal precedente o sucesiva, respectivamente.

Retornando a los ejemplos 11-14, aun si este sujeto produce de manera consistente la lateral en posición final de palabra (11), recurre ya sea a la sustitución (/1/ en 14) o al alargamiento (12) para expresar las líquidas ante consonante. Este último tratamiento, asimismo encontrado en posición final de palabra, implica el alargamiento de la vocal previa o bien la consonante subsecuente (12). Obviamente, el alargamiento compensatorio de la consonante que sucede a la líquida es exclusivo de las consonantes que ocurren antes de consonante. Las formas presentadas arriba, en las cuales tiene lugar la reducción o supresión de la líquida, exhiben la coexistencia de dicho proceso con el alargamiento de la vocal inmediatamente precedente en la representación fonológica, como puede apreciarse en el dato 12, que procede del mismo niño. En el caso de 17 (Tabla 4), el alargamiento de la vocal precedente tiene lugar ante consonante, como parte de un grupo heterosilábico.

\section{Tabla 4. Comportamiento de las líquidas ante consonante}

\begin{tabular}{ccccc}
\hline & Forma interna & Forma externa & Glosa & Edad \\
\hline 15 & /sól/ & {$[$ [̌ó:] } & [ma:] & $2 ; 0.20$ \\
\hline 16 & /mar/ & {$[\mathrm{ma}:]$} & 'mar' & $2 ; 0.20$ \\
\hline 17 & /un\#golpe/ & [um\#bó:pe] & 'un golpe' & $3 ; 11.18$ \\
\hline
\end{tabular}

Fuente: Elaboración propia.

El alargamiento de uno de los sonidos adyacentes para compensar la elisión de la líquida a final de palabra (15 y 16) y ante consonante (17), tiene como propósito preservar fonéticamente la duración silábica. Se ha comprobado, mediante estudios interlingüísticos, que este tratamiento es exclusivo de las consonantes en coda silábica, dado que el arranque no comparte la mora (Hayes, 1989; Kawahara, 2000).

Regresando ahora a los grupos homosilábicos, podemos decir que éstos constituyen los contextos más fuertemente marcados en la adquisición de la fonología del español, puesto que los segmentos adquiridos en cualquier otra posición, específicamente [1] y [r], se hacen extensivos a este contexto sólo tras un largo período de tiempo. Examinemos los datos de la Tabla 5. 
Tabla 5. Comportamiento de las líquidas en grupos homosilábicos (4;0.16)

\begin{tabular}{cccc}
\hline & Forma interna & Forma externa & Glosa \\
\hline 18 & /globo/ & {$[$ góßo] } & 'globo' \\
\hline 19 & /swéter/ & {$[\theta$ wétel] } & 'sweater' \\
\hline 20 & $/$ pólbo/ & {$[$ pólßo] } & 'polvo' \\
\hline 21 & $/$ kórto/ & /kólto/ & 'corto' \\
\hline 22 & /kokodrílo/ & {$[$ kokoðílo] } & 'cocodrilo' \\
\hline
\end{tabular}

Fuente: Elaboración propia.

Si bien /l/ ha sido adquirida en grupos heterosilábicos $(46,47)$, ni /l/ ni /r/ ocurren en grupos homosilábicos, lo cual parece motivado por diferencias en la relación de coordinación o transición implicada en ambos tipos de grupos en español. Así, mientras los grupos homosilábicos se traslapan (overlap) en gran medida - aunque no completamente, como se discutirá más adelante- los grupos heterosilábicos que contienen líquidas, se traslapan en menor grado. En otras palabras, además de la relación de transición (phasing) que cada consonante del grupo mantiene con la siguiente vocal, los grupos en arranque silábico implican un mayor grado de cohesión entre las consonantes del grupo, de tal manera que la relación de transición $\mathrm{C}$ - $\mathrm{C}$ es presumiblemente más fuerte que la relación de transición C-V (Browman \& Goldstein, 1990, citado en Gafos, 2002, p. 280). La justificación parece residir en la preservación de la recuperabilidad de ambas consonantes, evitando un grado de traslape que obscurecería una de las dos (Browman \& Goldstein, 1990, citado en Gafos, 2002, p. 281). En otras palabras, si bien los grupos del arranque implican un alto grado de traslape, no pueden exceder cierto límite, dado que la recuperabilidad estaría en riesgo. Ello difiere de los grupos heterosilábicos, si consideramos que la consonante de la coda exhibe una mayor relación con la vocal precedente y, presumiblemente, una relación más débil con la consonante subsecuente en comparación con el grupo que ocurre en el arranque. Recordemos que, al igual que las vocales, las consonantes en coda silábica son de carácter moraico (Hayes, 1989), y esta relación compartida es constatada secuencialmente por el alargamiento compensatorio. En este sentido, dado que los arranques son universalmente no moraicos, la supresión de una de las consonantes no induce, generalmente, al alargamiento compensatorio (Hyman, 1985 y McCarthy \& Prince, 1986 citados en Kawahara, 2000), como lo comprueban mis datos. 
Los grupos consonánticos que contienen líquidas varían en su comportamiento dependiendo de su carácter homosilábico o heterosilábico. En el primer caso, la segunda consonante del grupo, es decir, la líquida, se sustituye o se elide.

En 23-25 (Tabla 6) las líquidas /1/ y /r/, que son parte del grupo homosilábico, se suprimen, mientras que la primera consonante del grupo, o sea, la no-resonante, se preserva.

Tabla 6. Comportamiento de las líquidas en grupos homosilábicos (2;11.12)

\begin{tabular}{cccc}
\hline & Forma interna & Forma externa & Glosa \\
\hline 23 & /plánča/ & [pánča] & 'plancha' \\
\hline 24 & /pólbo/ & {$[$ póßo] } & 'polvo' \\
\hline 25 & /bárko/ & {$[$ báko] } & 'barco' \\
\hline
\end{tabular}

Fuente: Elaboración propia.

En contraste, en 26 y 27, que contienen grupos heterosilábicos, es la líquida - es decir, la consonante que funciona como coda - la que se suprime, en tanto que el arranque se mantiene. En este tipo de grupos es, pues, la primera consonante la que tiende a ser afectada. Si el grupo está constituido por líquida más consonante, es la primera la que se sustituye o suprime, independientemente de las características fonéticas del segmento subsecuente.

La misma observación es procedente en los grupos intersilábicos constituidos por líquida más no-resonante, como sucede en los ejemplos de la Tabla 7, en los que ya sea $/ \mathrm{l} / \mathrm{o} / \mathrm{r} /$ son afectados.

Dicho tratamiento parece relacionarse con la preferencia por las consonantes únicas que por los grupos consonánticos (Steriade, 1995).

Así, la extensión gradual a un número cada vez mayor de contextos parece concordar con las restricciones de marcaje identificadas interlingüísticamente. Los segmentos se adquieren antes en posiciones más naturales, mientras que los mismos segmentos se dominan más tardíamente en posiciones menos naturales. 
Tabla 7. Comportamiento de las líquidas en grupos heterosilábicos

\begin{tabular}{|c|c|c|c|c|}
\hline & Forma interna & Forma externa & Glosa & Edad \\
\hline 26 & /golpe/ & [gópe] & g'árbol' 'golpe' & $\begin{array}{c}2 ; 0.3 \\
2 ; 8.25 \\
3 ; 3.2 \\
4 ; 2.21\end{array}$ \\
\hline 27 & /árbol/ & [áßol] & 'árbol' & $\begin{array}{l}2 ; 8.25 \\
2 ; 1.12 \\
3 ; 9.14 \\
3 ; 11.18\end{array}$ \\
\hline
\end{tabular}

Fuente: Elaboración propia.

\section{4 Procesos fonológicos de las líquidas}

Como lo evidencian los datos expuestos arriba, el tratamiento en cada contexto varía en cierta medida desde un punto de vista cuantitativo, puesto que la posición intervocálica implica elisiones menos frecuentes que otros contextos - si bien los datos exhiben también casos de elisión intervocálica- dicho en otros términos, las sustituciones intervocálicas se presentan con más frecuencia que las elisiones. Por otra parte, la coda es un contexto favorable para el alargamiento ya sea de la vocal precedente o de la consonante subsecuente, así como para la supresión.

Además de estas tendencias, fonéticamente sustentadas, los estudios realizados en diferentes lenguas indican la existencia de variaciones intersubjetivas (Ferguson \& Slobin, 1973; McLeod, Van Doorn \& Reed, 2001 y todos los trabajos mencionados arriba referentes a la adquisición de la fonología del español), de tal modo que dos individuos pueden tratar de manera diferente la misma unidad léxica. Por ejemplo, algunos niños cuyos datos se han mostrado arriba recurren al alargamiento compensatorio para preservar la cuenta moraica (mora count), en tanto que otros prefieren la supresión. Pero los datos hacen, asimismo, patente, que formas que exhiben diferentes "estrategias" o diferentes estadios de desarrollo fonológico coexisten en el mismo individuo; e incluso la misma palabra puede recibir distintos tratamientos. Por ejemplo, los mismos sujetos pueden elidir un segmento específico en una unidad léxica, y sustituir el mismo segmento en otra.

La sustitución de las líquidas por semiconsonantes $(y, w)$ - lo que se conoce en inglés como "gliding" (Dale, 1976) — se encuentra ampliamente. En el caso del español, se prefiere $[y]$ en todos los contextos. 
Hasta este momento, he presentado ejemplos de sustitución intervocálica y en posición final de palabra. Los datos de la Tabla 8 exhiben el mismo patrón sustitutivo antes de consonante al interior de palabra.

Tabla 8. Procesos sustitutivos de las líquidas ante consonante

\begin{tabular}{ccccc}
\hline & Forma interna & Forma externa & Glosa & Edad \\
\hline 28 & /árbol/ & [áyßol] & 'árbol' & $2 ; 8.8$ \\
\hline 29 & /barkíjo/ & [bayikíyo] & 'barquillo' & $2 ; 8.8$ \\
\hline * Eblen emplea el símbolo [i] para el sonido semiconsonántico que yo represento como [y]. \\
Fuente: Elaboración propia.
\end{tabular}

Además de la sustitución, la elisión del grupo consonántico se presenta en las etapas más tempranas del desarrollo de los grupos homosilábicos. Dicho proceso implica la supresión de los dos elementos del grupo consonántico, como en la forma [íyo], producida por uno de los sujetos de Eblen para la palabra /gríjo/ "grillo". Este tratamiento se encuentra con mayor frecuencia a inicio de palabra que en interior de palabra, donde los grupos son o bien simplificados o sólo parcialmente suprimidos.

La lateral es el primer miembro que aparece. Una vez que ello sucede, la líquida emergente actúa como realización fonética no sólo de la lateral, sino de la clase en su conjunto. Los datos previos muestran que [1] no es sólo la realización de /1/, sino también de /r/. Los datos de la Tabla 9 ejemplifican la sustitución de las vibrantes simple y múltiple por [1] en posición intervocálica.

Tabla 9. Comportamiento de las vibrantes

\begin{tabular}{ccccc}
\hline & Forma interna & Forma externa & Glosa & Edad \\
\hline 30 & /péřo/ & [pelo] & 'perro' & $3 ; 9.14$ \\
\hline 31 & $/$ periko/ & [pelíto] & 'perico' & $3 ; 9.14$ \\
\hline \multicolumn{5}{c}{ Fuente: Elaboración propia. }
\end{tabular}

En los datos de la Tabla 1o la sustitución tiene lugar en arranque complejo. 


\section{Tabla 10. Comportamiento de las vibrantes en grupos consonánticos homosilábicos $(2 ; 11.9)$}

\begin{tabular}{cccc}
\hline & Forma interna & Forma externa & Glosa \\
\hline 32 & $/$ krema/ & {$[$ kléma] } & 'crema' \\
\hline 33 & $/$ bráso/ & [bláӨo] & 'brazo' \\
\hline 34 & $/$ frúta/ & [fúta] & 'fruta' \\
\hline
\end{tabular}

Fuente: Elaboración propia.

El proceso sustitutivo se hace extensivo a diferentes contextos al interior de la sílaba y la palabra, de conformidad con el orden expuesto arriba; de tal modo que en el caso de los grupos homosilábicos la sustitución tiene lugar mucho después que en otros contextos de la palabra. Entre la adquisición de /1/ y la adquisición de la siguiente líquida transcurre un largo período de tiempo, el cual permite la adquisición de otro sonido sonoro que comparte el mismo punto de articulación de la líquida. Dicho sonido es [d] o [ð], dependiendo del contexto fonético específico. Como lo hace notar Amador (1978), la elección de la oclusiva o de la fricativa por el niño, corresponde a la variación alofónica del fonema /d/, según la cual, la oclusiva ocurre en posición inicial y después de /1/ y /n/; en tanto que la fricativa [ð] ocurre en posición intervocálica y después de consonante heterorgánica. Una vez que $[\mathrm{d}]$ y [ð] se encuentran disponibles en el repertorio fonético del niño, se convierten en la elección predilecta para $/ \mathrm{r} / \mathrm{y} / \check{r} /$ en posición intervocálica $\mathrm{y}$, en algunos niños, también a final de palabra. En este último caso se realiza como el correspondiente segmento sordo $[\Theta]$ o la oclusiva $[\mathrm{t}$ ], en congruencia con la pronunciación adulta de /d/ en posición preconsonántica y final de palabra. Los datos de la Tabla 11 ilustran dicho comportamiento. 
Tabla 11. Comportamiento de las vibrantes en diferentes contextos

\begin{tabular}{|c|c|c|c|c|}
\hline & Forma interna & Forma externa & Glosa & Edad \\
\hline & \multicolumn{4}{|c|}{$\mathbf{v}_{---} \mathbf{v}$} \\
\hline \multirow{3}{*}{35} & /naríz/ & [naðí $\theta]$ & 'nariz' & $2 ; 8.8$ \\
\hline & & & Mañ & $2 ; 11,12$ \\
\hline & \multicolumn{4}{|c|}{ \#_ } \\
\hline \multirow[t]{2}{*}{36} & /řepálo/ & [depálo] & 'regalo' & $2 ; 8.8$ \\
\hline & \multicolumn{4}{|c|}{ _-___\# } \\
\hline 37 & /Čór/ & [čó $\theta$ ] & 'short' & $3 ; 1.16$ \\
\hline 38 & /mar/ & [mát] & 'mar' & $5 ; 8,3$ \\
\hline 39 & /alfiler/ & [af:ilét] & 'alfiler' & $4 ; 7.7$ \\
\hline
\end{tabular}

Fuente: Elaboración propia.

Como Eblen (1980, p. 171) lo hace notar, si bien [d] y [ð] se encuentran ya disponibles en el repertorio infantil, [1] continúa reemplazando, de manera alterna y por algún tiempo, a las dos no-laterales. La frecuencia en la sustitución de esta última decrece de manera consistente conforme el desarrollo fonológico avanza.

La sustitución por [d] de las líquidas no laterales ha sido considerada altamente específica del español, puesto que sólo de manera ocasional se ha documentado en otras lenguas (Anderson \& Smith, 1987; Stoel, 1974).

Por lo que respecta al español, el papel sustitutivo de $[d]$ y $[ð]$ con relación a las líquidas no laterales persiste hasta la adquisición de la vibrante simple, que asume el papel de la oclusiva sonora como sustituto de la vibrante múltiple.

El último paso en la adquisición de las líquidas lo constituye la incorporación de la vibrante múltiple al inventario fonético del niño. Si bien diferentes formas fonéticas son usadas por el mismo niño para la misma unidad léxica o para alguna similar, la frecuencia de las formas que incluyen la vibrante múltiple se incrementa conforme el niño crece.

Respecto al tratamiento de los segmentos líquidos restringidos, apreciamos que las elisiones son más frecuentes en los grupos homosilábicos y en posición inicial en los estadios tempranos del desarrollo. En cambio, los segmentos ubicados en las posiciones preconsonántica y final de palabra, pueden ser elididas o "compensadas" mediante alargamiento compensatorio ya sea de la vocal o de la consonante adyacente, en la forma superficial. 
Las sustituciones suceden, a menudo, en el contexto intervocálico, donde las elisiones son raras. $\mathrm{Al}$ ampliarse los recursos fonéticos del niño, las sustituciones se tornan más frecuentes y hacen posible producir un número creciente de formas fieles (faithful forms).

En los estadios más tempranos del desarrollo de las líquidas identificados en la muestra, todas las ocurrencias de líquidas se encuentran restringidas con excepción de la posición intervocálica. Como he argumentado, dicho comportamiento puede obedecer a restricciones posicionales que afectan a segmentos o rasgos específicos; es decir, el contexto más natural para las líquidas parece ser la posición intervocálica, así como para otras clases de sonidos, como las oclusivas sonoras.

Esta situación, en la que algunos rasgos se ven favorecidos por el contexto circundante, se ha identificado en muchas lenguas. En español, la posición intervocálica favorece el pleno contraste no sólo entre los fonemas líquidos, sino también entre las nasales, que en otros contextos tienden a ser neutralizadas o limitada, o limitadas la ocurrencia de sus miembros. Por ejemplo, /r/ y /ñ no ocurren en posición inicial, pero sí entre vocales. Conforme las líquidas emergen a la superficie tanto en posición intervocálica como inicial, las restricciones susceptibles al contexto afectan sólo a las líquidas en los restantes contextos léxicos. El alcance de estas restricciones se reduce gradualmente conforme la adquisición de las líquidas se hace extensiva a un mayor número de contextos en la palabra.

Etapas posteriores en la adquisición de la fonología involucran limitaciones sucesivas en el alcance de las restricciones, hasta que las restricciones en la producción de dos consonantes se superan en dicho contexto. Sin embargo, como se mencionó anteriormente, es posible encontrar dos tratamientos distintos no sólo en diferentes sujetos, sino incluso en el mismo, ya sea con relación a diferentes unidades léxicas o a la misma palabra. Las evidencias empíricas muestran (Eblen, 1980; McLeod et al., 2001) que ésta es una característica de la adquisición de la fonología en general, indistintamente de la lengua en cuestión.

En virtud de que la substitución por la oclusiva sonora de los segmentos no laterales de la clase de las líquidas ha impactado a los estudiosos del proceso de adquisición como una característica específica del español, me gustaría profundizar en la interpretación que acabo de ofrecer. En mi perspectiva, la preferencia por [d] y $[ð]$ con respecto a la lateral una vez que ambos sonidos se encuentran disponibles para quien adquiere el español, implica la conjunción, en el mismo segmento, del punto de articulación, el valor positivo para el rasgo de sonoridad, y la dirección de 
la corriente del aire. Es decir, si bien [1] comparte con /r/ y con / $\check{\mathrm{r}} /$ todos los rasgos del modo de articulación, difiere en la dirección del aire, puesto que el aire sale lateralmente a través de la cavidad oral. Así, pues, asumo que una vez que un sonido no lateral sonoro homorgánico diferente de las nasales homorgánicas se encuentra disponible para el niño, se convierte en mejor candidato que la lateral, al menos hasta que, para el caso de la vibrante múltiple, la vibrante simple asume más tarde dicho papel.

El curso del desarrollo expuesto a lo largo del presente trabajo, se basa en la posición de los sonidos líquidos al interior de la sílaba o la palabra. Una explicación plena y empíricamente fundamentada de la adquisición de las líquidas no puede alcanzarse hasta examinar la influencia del contexto fonético en las líquidas, así como de éstas en los segmentos adyacentes. Si bien las líquidas obedecen a las restricciones "posicionales" esbozadas arriba, el segmento específico que actúa como sustituto de aquéllas depende de la naturaleza de los sonidos que concurren en la misma secuencia. Este importante aspecto en el desarrollo de la clase de sonidos que nos ocupa, será objeto de un estudio ulterior.

\section{Niños con desarrollo atípico}

En el caso de los niños que presentan desarrollo fonológico atípico, encuentro que los procesos en sí no difieren - salvo en casos específicos en los que el niño aún no cuenta con los dientes frontales y tiende a usar otros que tengan un punto de articulación diferente, como en el caso de [gren] por "tren”. Lo que sí se encuentra es un cierto retraso en la adquisición de la representación fonética de algunos fonemas.

La valoración fonológica con otros niños con desarrollo atípico indica que la lateral / / es la primera en adquirirse en todos los contextos, en contexto intervocálico, inicial y final; en tanto que los grupos consonánticos homosilábicos y heterosilábicos se adquieren con posteridad. Eventualmente, se suprime en grupo consonántico homosilábico, como en [igésya] "iglesia" y produce un efecto asimilatorio sobre otras consonantes líquidas (por ejemplo, [kokolílo] "cocodrilo”) y /d/ como en [salíla] "salida”, cuando éstas ocurren en contextos adyacentes.

La vibrante se adquiere primero en posición intervocálica y posteriormente a final de palabra. En grupos consonánticos homosilábicos y heterosilábicos y en posición final de palabra tiende a suprimirse, como en: [poké] "por qué”, [peðí] 
“perdí", [myékoles] “miércoles”; [pyéða] “piedra”, [Өémpe] "siempre”, [fixól] “frijol"; [kokolílo] "cocodrilo".

De acuerdo con mi experiencia como terapeuta del lenguaje, en el caso de la vibrante múltiple, que es la de más tardía adquisición dentro de las líquidas, se procede a enseñar al niño a emitir una vibrante múltiple bilabial, asociándola con el sonido producido por el motor de una motocicleta; de modo que estén conscientes del modo de producción y, posteriormente, puedan realizarlo en posición alveolar. En el caso de los grupos consonánticos heterosilábicos, especialmente los integrados por una consonante más la vibrante simple, en el caso de uno de los niños se incluye la vocal inmediatamente posterior al grupo consonántico (por ejemplo, "plato" como [paláto] y "tren" como [terén]) y se pide a los niños decirlo cada vez más rápido hasta que la vocal se suprime y logran articular [pláto] y [tren], respectivamente. Estas estrategias han resultado relevantes para mejorar gradualmente su articulación.

A nivel educativo, el desarrollo fonológico atípico puede implicar que el niño se inhiba con pares y adultos. Según Moreno y Mateos (2006), la dificultad articulatoria puede ejercer impacto en la personalidad del niño. Las actitudes y reacciones de un niño pueden ser muy diversas tanto en el entorno familiar como en el social y escolar, al que debe hacer frente. El niño puede sentirse marginado en virtud de su dificultad articulatoria, o él mismo puede marginarse aislándose de los demás debido a su desvalorización, ya que evitando el contacto con cualquier persona de su entorno más o menos cercano, logra reducir el impacto que le genera la interacción comunicativa.

Actualmente, la tendencia es que los docentes participen en la socialización del niño y que sean mediadores de lo que éste quiere comunicar oralmente, y asesoren a la familia para que el niño supere sus limitaciones fonéticas. En tal sentido, Murguía, Hernández y Carrera (2016) mencionan la relevancia de la instrumentación de acciones a partir de las cuales la educación llegue a todos los alumnos en contextos regulares y no segregados, aceptando diferencias, la diversidad, y motivando el trato equitativo de cada alumno. En este contexto, el logopeda, con el propósito de ofrecer una educación de calidad, ha de coordinarse con los maestros y la familia. 


\section{Conclusiones}

En el presente artículo he abordado la adquisición de las líquidas desde la perspectiva de la fonología fonéticamente orientada. Consecuentemente, me he centrado en la motivación fonética de la adquisición de las líquidas del español a partir de a) la evidencia interlingüística y b) de la aportada por los estudios sobre adquisición del lenguaje, la mayoría de ellos de índole articulatoria.

La interacción de diferentes tipos de restricciones (tanto de naturaleza segmental como secuencial) que influyen y configuran la producción del niño, ha sido asimismo discutida. Sobre esta base, se han explicado las diferentes estrategias puestas en juego por distintos individuos. Junto con las tendencias interlingüísticamente documentadas y la evidencia aportada por los estudios sobre adquisición, los patrones fonotácticos específicos del español desempeñan un papel en la adquisición de las líquidas, ya sea a nivel de producción o de forma subyacente. Constituyen también un factor relevante en la distribución de los sonidos-sustituto y en el retraso comparativo en la adquisición de sonidos específicos, tales como [d] o [ð].

Se identificaron dos tipos de restricciones cuya interacción configura el proceso de adquisición de las líquidas en español: restricciones segmentales y restricciones sensibles al contexto. Como parte de este último, se encontraron dos tipos de contexto relevantes: la posición de las líquidas al interior de las palabras, y las propiedades fonéticas de los segmentos circundantes, especialmente los adyacentes.

Atendiendo a esta interacción, se obtuvo un cuadro más completo del proceso de adquisición de este tipo de sonidos, en comparación con otros estudios que circunscriben sus alcances a contextos específicos (Anderson \& Smith, 1987; Eblen, 1980; Stoel, 1974, entre otros).

Asimismo, se identificó el orden en el que tienden a adquirirse las líquidas, así como los contextos a los que gradualmente se hacen extensivas las adquisiciones segmentales. Se discutió, de igual manera, qué segmento o secuencia actúa como sustituto y la influencia de los sonidos circundantes que constituyen la secuencia en su conjunto, especialmente en las etapas más tempranas. Conforme el desarrollo fonológico avanza, dichas influencias contextuales ejercen cada vez menos impacto en la elección del niño y permiten la emergencia de diferentes combinaciones de sonidos en la misma secuencia. Desde esta perspectiva he discutido, por un lado, las relaciones entre líquidas y semiconsonantes y, por otro, entre líquidas y dentales sonoras no-resonantes, no sólo en el plano segmental, sino también en el secuencial. Si bien 
estudios previos han señalado la interacción entre líquidas y otros sonidos y grupos consonánticos, ésta no se había discutido en su complejidad.

Se discutió la posibilidad de que existan diferencias, en casos y aspectos específicos, entre la representación fonológica del niño y la del adulto, especialmente el impacto que ejercen procesos tales como el reanálisis y la analogía tanto en la representación fonológica como en la realización fonética del niño. Algunas unidades léxicas cuyo comportamiento parece obedecer a tratamientos idiosincrásicos y requerir la postulación de restricciones específicas, se comprenden fácilmente si el enfoque del análisis se amplía para considerar la interacción con otros niveles lingüísticos.

En el caso de los niños que presentan desarrollo atípico, salvo en el caso mencionado en que no se cuenta con los dientes frontales, el proceso de adquisición de las líquidas y los procesos fonológicos empleados no difieren de los aplicados por niños con desarrollo típico. Lo que se encuentra es un retraso en la adquisición de la representación fonológica de algunos fonemas o a reducir el número de sílabas de la unidad léxica. En tales casos, se sugiere aumentar gradualmente el número de sílabas en palabras esdrújulas, ya que la sílaba acentuada tiende a conservarse.

Es importante, en el ámbito educativo y escolar, en general, proporcionar la terapia correspondiente. Actualmente esta necesidad se está atendiendo a través de instituciones como el Centro de Atención Psicopedagógica de Educación Especial (CAPEP) y de la Unidad de Servicio de Apoyo a Educación Regular (USAER).

\section{Lista de Referencias}

Amador, M. (1978). Fonología del Español hablado por cuatro niños de dos a tres años (Tesis de maestría inédita). Escuela Nacional de Antropología e Historia, México.

Anderson, R., \& Smith, B. (1987). Phonological development of two-year-old Monolingual. Puerto Rican Spanish-speaking children. Journal of Child Language, 14, 57-78.

Bakovic, E. (2001). Nasal place neutralization in Spanish. University of Pennsylvania Working Papers in Linguistics, 7, 1-12.

Bernhardt, M., Hanson, R., Pérez, D., Ávila, C., Lleó, C., Stemberger, J. P., Carballo, G., Mendoza, E., Fresneda, D., \& Chávez-Peón, M. (2015). Word structures of Granada Spanish-speaking preeschooler with typical versus protracted pho- 
nological development. International Journal of Language and Communication Disorders, 50(3), 298-311.

Bosch, L. (1984). El desarrollo fonológico infantil: una prueba para su evaluación. En M. Siguan (Ed.), Estudios sobre psicología del lenguaje infantil (pp. 33-56). Madrid: Pirámide.

Bradley, T. G., \& Schmeiser, B. S. (2003). On the phonetic reality of / $/$ in Spanish complex onsets. En P. M. Kempchinsky \& C. E. Pineros (Eds.), Theory, Practice and Acquisition (pp. 1-20). Massachusetts: Cascadilla Press.

Braine, D.S. (1976). Reseña de "The Acquisition of Phonology: A Case Study" por Neilson V. Smith. Language, 52, 489-498.

Crystal, D. (1987). The Cambridge Encyclopedia of Language. Cambridge: Cambridge University Press.

Dale, P. S. (1976). Language development: structure and function. Seattle: University of Washington/Holt, Rinehart and Winston.

Eblen, R. E. (1980). Phonological processes in the development of liquids in Mexican children. En R. S. Maller (Ed.), Papers from the 1979 Md. American Linguistics Conference (pp.168-174). Lincoln: University of Nebraska.

Farnetani, E. (1997). Coarticulation and connected speech processes. En W. Hardcastle \& J. Laver (Ed.), The handbook of phonetic sciences (pp. 371-404). Cambridge: Blackwell.

Fee, E. J. (1995). Segments and syllables in early language acquisition. En J. Archibald (Ed.), Phonological acquisition and phonological theory (pp. 43-61). Nueva Jersey: Lawrence Erlbaum.

Ferguson, C. A., \& Slobin, D. I. (Eds.). (1973). Studies of child language development. Nueva York: Holt, Rinehart and Winston.

Fontanella, M. B. (1981). Adquisición fonológica en español bonaerense. Bahía Blanca, Argentina: Universidad Nacional del Sur.

Gafos, A. (2002). A grammar of gestural coordination. Natural Language and Linguistic Theory, 20, 269-337.

González, A. (1981). Perspectivas Teóricas sobre adquisición del lenguaje. Recuperado de https://www.researchgate.net/publication/266873795_PERSPECTIVAS TEORICAS SOBRE_LA_ADQUISICON_DEL_LENGUAJE

Harris, A., \& Campbell, L. (1995). Overview of a theory of syntactic change. En A. Harris \& L. Campbell, Historical Syntax in Cross Linguistic Perspective (pp. 4995). Cambridge: University Press. 
Hayes, B. (1989). Compensatory lengthening in moraic phonology. Linguistic inquiry, 20, 253-306.

Hayes, B. (1997). Phonetically driven phonology: the role of optimality theory and inductive grounding. Proceedings. Milwakee Conference on Formalism and Functionalism in Linguistics.

Hopper, P., \& Traugott, E. (2000). Grammaticalization. Cambridge: University Press. Ingram, D. (1976). Phonological analysis of a child. Glossa, 10, 3-27.

Jun, J. (1995). Place assimilation as the result of conflicting perceptual and articulatory constraints. Proceedings of the West Coast Conference on Formal Linguistics, 14.

Kawahara, S. (2000). Similarity among variants: output-variant correspondence (Tesis de bachillerato inédita). International Christian University. Recuperada de http://user.keio.ac.jp/ kawahara/pdf/ov faith.pdf

Kühnert, B., \& Nolan, F. (1999). The origin of coarticulation. En W. Hardcastle \& N. Hewlett (Eds.), Coarticulation. Theory, data and techniques (pp. 7-30). Cambridge: University Press.

Lleó, C., \& Prinz, M. (1996). Consonant clusters in child phonology and the directionality of syllable structure assignment. Journal of child language, 23(1), 31-56.

Lleó, C., \& Prinz, M. (1997). Syllable structure parameters and the acquisition of affricates. En S. J. Hannahs \& M. Young-Scholten (Eds.), Focus on phonological acquisition (pp. 143-164). Amsterdam: John Benjamins.

Macken, M. A. (1975). The acquisition of intervocalic consonants in Mexican Spanish: a cross-sectional study based on imitation data. Papers and reports on child language development, 9, 24-42.

Macken, M.A. (1978). Permitted complexity in phonological development: one child's acquisition of Spanish consonants. Lingua, 44, 219-253.

Macken, M. A., \& Barton. D. (1980). A longitudinal study of the acquisition of the voicing contrast in American-English word-initial stops, as measured by voice onset time. Journal of Child Language, 7, 41-74.

Maez, L. F. (1981). Spanish as a first language: the early stages (Tesis doctoral inédita). University of California, Santa Bárbara.

McLeod, S., Van Doorn, J., \& Reed, V. A. (2001). Consonant cluster development in two-year-olds: general trends and individual differences. Journal of Speech Language and Hearing Research, 44(5), 1144-1172.

Menn, L. (1978). Phonological units in beginning speech. En A. Bell \& J. B. Hooper (Eds.), Syllables and segments (pp. 157-172). Amsterdam: North Holland. 
Moreno, J., \& Mateos, R. (2006). Personalidad del niño y dislalia. Infocop On-Line. Murguía, M., Hernández, T., \& Carrera, M. (2016). El rol de la logopedia en la inclusión educativa con escolares con trastorno de la comunicación oral. Avances en Supervisión Educativa, 26. doi:https://doi.org/10.23824/ase.voi26.574

Oropeza, M. (1987). El curso del desarrollo consonántico en niños de dos a seis años que adquieren el español como lengua materna (Tesis de Licenciatura inédita). Universidad Veracruzana, México.

Oropeza, M. (200o). Factores lingüísticos que inciden en el desarrollo fonológico: Reflexiones a partir de un estudio con niños de dos a seis años que adquieren el español como lengua materna. Revista Lingüística Mexicana, 1(2), 229-246.

Oropeza, M. (2001a). Procesos fonológicos en la adquisición del español como lengua materna. En C. Rojas \& L. de León (Coords.), La adquisición de la lengua materna. Español, lenguas mayas, euskera (pp. 125-140). México: Universidad Nacional Autónoma de México/Centro de Investigación y Estudios Superiores en Antropología Social.

Oropeza, M. (julio-diciembre, 2001b). Adquisición de la estructura silábica del español en niños de dos a seis años. Colección Pedagógica Universitaria, 36, 80-92.

Oropeza, M. (julio-diciembre, 2012). Variación, reanálisis y analogía en la adquisición de la fonología del español, CPU-e, Revista de Investigación Educativa, 15, 27-47. Recuperado de http://revistas.uv.mx/index.php/cpue/article/view/18

Raymond, G. (2017). Consonant acquisition in typically developing preschool speakers of Granada Spanish (Tesis de maestría inédita). Universidad de British, Columbia, Canadá.

Smith, C. (1993). Prosodic patterns in the coordination of vowel and consonant gestures. En B. Connell \& A. Arvaniti (Eds.), Phonology and phonetic evidence. Papers in laboratory phonology IV (pp. 205-234). Cambridge: University Press.

Steriade, D. (1995). Phonetics in phonology: The case of laryngeal neutralization (Manuscrito no publicado). Departamento de Lingüística, Universidad de California, Los Ángeles.

Steriade, D. (2001). Directional asymmetries in place assimilation. A perceptual account. En E. Hume \& K. Johnson (Eds.), The role of speech perception in phonology (pp. 219-250). San Diego: Academic Press.

Stoel, C. M. (1974). The acquisition of liquids in Spanish (Tesis doctoral inédita). California: Stanford University.

Timberlake, A. (1977). Reanalysis and actualization in syntactic change. En C. N. Li 
(Ed.), Mechanisms of syntactic change (pp. 141-180). Austin: University of Texas Press.

Velleman, S., \& Vihman, M. (2002). The optimal initial state. Recuperado de http:// roa.rutgers.edu/files/552-1002/552-1002-VELLEMAN-0-0.PDF

Vihman, M. M. (1981). Phonology and the development of the lexicon: Evidence from children's errors. Journal of Child Language, 8, 239-264.

Westbury, J., \& Keating, P. (1986). On the naturalness of stop consonant voicing. Journal of Linguistics, 22, 145-166.

Zuluaga De, T. (1981). Procesos en la sistematización de los sonidos del español en niños de corta edad. Language and Language Behavior Abstracts, 15(4), 776. 